\title{
LES INTELLECTUELS CAMEROUNAIS SOUS LE REGIME AHIDJO (1958-1982)
}

\author{
Maximin EMAGNA ${ }^{1}$ \\ s/c IISA, rue Defacqz, 1 , Bte 11 \\ B-1000 Bruxelles
}

\section{SUMMARY}

\section{THE INTELLECTUALS IN CAMEROON UNDER THE RULE OF AHIDJO} (1958-1982)

A study concerning the intellectuals of the Third World in general, and African intellectuals in particular, should not exclude the political context in which intellectuals live and work. Indeed, democracy cannot truly emerge and remain durable without the existence of freedom of thought and criticism.

In Cameroon, intellectuals and their ideas have always been considered seditious and subversive. For this reason, they have often been persecuted. Some have chosen freely or by the force of circumstances to become bureaucrats within the administrative and political structures of the country to ensure their survival (political and social immortality) as also that of their family. Others, by principle, have combined their intellectual and cyclical factors which push intellectuals either to become civil servants or to choose exile. It also contradicts the idea of intellectual deficiency in Africa. Indeed, some categories of intellectuals such as writers, via their writings, ask questions and provide a critical review of the

1. Chargé de recherches à l'Institut International des Sciences Administratives (Bruxelles). Membre de l'équipe de recherche du Centre universitaire de recherches administratives et politiques de Picardie (CURAPP-CNRS, Amiens, France) et Professeur invité de Gestion des affaires publiques au CIDEP/Nations-Unies de l'Université catholique de Louvain (Belgique) de 1993 à 1995. 
economic, political and social reality of the country. They even propose alternative ideas and proposals to such questions as management, government and governance in the coutry. Dictatorships may destroy individuals, but never ideas or the spirit of liberty.

\section{RESUME}

Une réflexion sur les intellectuels du Tiers Monde en général, et sur les intellectuels africains en particulier ne devrait pas faire l'économie du contexte politique dans lequel ces derniers sont censés vivre et travailler. Et la démocratie ne peut véritablement émerger et s'instaurer durablement sans l'existence d'espaces de liberté, de réflexion et de critique.

Au Cameroun, les intellectuels et leurs idées ont toujours été taxés de séditieux et de subversifs. Pour cette raison, ils ont souvent été persécutés. Les uns ont choisi volontairement ou par la force des choses de devenir des bureaucrates en s'intégrant dans les structures administratives et politiques du pays afin d'assurer leur survie (immortalité politique et sociale) ainsi que celle de leur famille. D'autres, par principe, ont allié le combat intellectuel et la lutte armée. Le rapport de force a contraint bon nombre d'entre eux à l'exil. Cet article, illustre les conditions structurelles et conjoncturelles qui conduisent à la fonctionnarisation des intellectuels ou les pousse à l'exil. Il contredit également l'idée d'un déficit intellectuel en Afrique. Car, certaines catégories d'intellectuels comme les écrivains, à travers leurs écrits, posent des questions et un regard critiques sur la réalité économique, politique et sociale. Ils vont même jusqu'à proposer des idées et des solutions alternatives aux problèmes de gestion et de gouvernement et de gouvernance que connaît le pays. La dictature tue les hommes, mais ne tue ni les idées, ni l'esprit de liberté.

On se pose souvent la question de savoir comment un pays comme le Cameroun qui compte de nombreux diplômés de haut niveau formés sur place, ou à l'étranger, n'arrive pas à s'écarter des voies tracées par le Colonisateur et à se défaire de toutes les formes de tutelle. Comment expliquer et comprendre que nombreux sont les intellectuels qui sont contraints de vivre en exil à l'étranger. Comment expliquer l'allégeance au poivoir et/ou démission ou la " trahison ${ }^{1}$ de bon nombre 
d'intellectuels? Quelle images les intellectuels africains donnent-ils aux générations de la " démocratie ", nées entre les années 70 et 80 ?. Pourquoi rappeler ce que tout le monde sait sans doute déjà sur les intellectuels africains: que par leur " exercice du droit de (co)propriété et de (co)gérance ${ }^{2}$ de l'Etat postcolonial, ils ont démissionné de leur mission et de leur rôle de producteur d'idées nouvelles sur les réalités sociales et politiques. Certains, vont même jusqu'à affirmer qu'il n'existe pas d'intellectuels en Afrique! Notre contribution tente de démontrer que les africains produisent des idées ${ }^{3}$, réfléchissent sur la société, pensent leur avenir, et suggèrent même des solutions alternatives, qu'il revient aux hommes politiques d'expérimenter et de mettre en pratique. L'expertise locale existe! Mais, alors que certains espéraient à juste titre, que "l'ère démocratique " des années 90 , permettrait la clarification du débat sur l'identité et le statut des intellectuels ${ }^{4}$ dans la société, on assiste malheureusement, dans un pays comme le Cameroun, à la " prostitution" des intellectuels par des hommes politiques.

Par ailleurs, il nous semble important de rappeler à la mémoire des nouvelles générations d'africains, celle des années 70 et 80 , les situations des intellectuels dans la lutte pour la construction de leur pays. Il est clair que la mémoire d'un pays est nécessaire aussi bien à sa jeunesse, qu'à la société entière. Notre analyse se veut une étude-bilan qui permet de comprendre la continuité d'une politique de repression ${ }^{5}$, repression qui contraint les intellectuels à la revolte, à l'errance ou à l'exil. Les intellectuels qui veulent utiliser à fond l'arme de l'interrogation et du questionnement permanent ${ }^{6} a$ fond, "ceux qui veulent rester des intellectuels libres avant tout et exercer leur liberté intellectuelle "7, à la révolte, à l'errance, ou à l'exil. Or, on constate néanmoins avec l'écrivain et opposant politique camerounais Mongo Beti que "...Tant de discours se font sur la pauvreté des pays sous-développés, tant d'invites à l'effort sont adressées à leurs habitants, et surtout à leurs élites, sommées de se sacrifier, de se crucifier pour leurs peuples, conspuées par la presse capitaliste internationale qui appelle "fuite des cerveaux" leur ultima ratio contre la dictature déshumanisante, l'exil" ${ }^{\prime}$. L'analyse de la question de la "fuite des cervaux" mérite d' être approfondie'.

De nos jours, une réflexion sur les intellectuels du Tiers Monde en général, et sur les intellectuels africains en particulier, ne devrait pas faire l'économie du contexte politique dans lequel ces derniers sont censés vivre et travailler. On se saurait dès lors comprendre le désarroi des intellectuels africains en exil en faisant l'économie du contexte socio politique et culturel qui les pousse à opter pour ce choix. Il nous semble que ce n'est pas par simple recherche d'un confort matériel que ces intellectuels "fuient" leur pays, il s'agit parfois, d'une "exil forcé "10. 
La présente analyse, qui emprunte à de nombreuses études sur le Cameroun, tente d'aborder la question des intellectuels en la resituant dans son contexte historique, politique, social et culturel. Nous tentons ici de répondre et d'expliquer les raisons qui ont poussé de nombreux auteurs à espérer un changement politique profond et radical au Cameroun dès 1982 avec l'accession de " l'intellectuel "Biya au pouvoir et le recours aux "nouveaux intellectuels" (les technocrates et les techniciens de haut niveau) ${ }^{11}$ dans les instances dirigeantes du pays. Il nous a semblé difficile de présenter la situation des intellectuels au Cameroun aujourd hui, sans un détour historique préalable à la période antérieure ${ }^{12}$. Et nous restons convaincu qu'aucune étude sur le Cameroun contemporain ne peut véritablement être menée en faisant l'impasse sur la période de la constitution de l'Etat colonial et surtout post colonial. Tout semble lié par le projet hégémonique du régime Ahidjo. Il y a comme un continum, une continuité historique qu'un chercheur ne saurait évacuer. Comment comprendre par exemple la réponse de Mgr Tumi, Cardinal camerounais, répondant à la question d'un journaliste: "... Et les intellectuels, parlent-ils ? Mgr Tumi répond: "Très peu, Ils n'osent pas. Ils ont peur [Peur de la répression]. Beaucoup sont des fonctionnaires. Ils ont donc peur de perdre leur place $\ldots{ }^{\prime 13}$. Notre article est dominé par ce sentiment de peur et d'insécurité que vivent les intellectuels africains, sentiment dépeint dans une pièce de théâtre de l'auteur à paraître sous le titre Afrique rocambolesque I: La Cité infernale. Nous nous limitons ici au bilan politique d'Ahidjo, notamment des rapports que ce dernier entretenait avec les milieux intellectuels. Notre analyse ne porte pas de jugement sur le bilan économique de cette période, qui par certains côtés ne semble pas avoir été globalement négatif ${ }^{14}$.

Il ne fait aucun doute qu'il existe des intellectuels au Cameroun, l'intellectuel entendu comme un producteur et un diffuseur d'idées sur la réalité sociale, économique et politique. Pour l'imagerie populaire, cette expression est vaste: on reconnaît ainsi que les universitaires, les "docta" ${ }^{15}$ (porteurs ou aspirant au titre de docteur), les enseignants, les journalistes, les écrivains, les étudiants, les "diplômés", font partie d'une catégorie particulière de la société: les "longs crayons" (ceux qui ont fait des études poussées, du niveau de l'enseignement supérieur, la plupart du temps). Faute de n'avoir pas pu vérifier cette conception par l'étude qui devait sous-tendre cet article, nous utiliserons le terme intellectuel soit pour de manière englobante, soit en spécifiant selon les cas lorsque nous faisons référence à une catégorie particulière. L'expression sera surtout utilisée ici pour désigner les écrivains, auteurs littéraires.

Nous nous proposons de revenir sur la période d'avant 1982 pour montrer comment le projet hégémonique du régime s'est constitué avec et contre les intellectuels(I). 
D'autre part, le régime de terreur et de peur instauré par la suite -bien que réprimant de manière particulièrement violente toute velléité d'opposition- a certes réduit la production d'essais politiques et en a limité la portée, mais a permis l'émergence d'une littérature "politique" au Cameroun, littérature marginale qui pose un diagnostic sans complaisance de la situation socio-politique du pays et, regorge de solutions alternatives, contredisant ainsi les discours sur le déficit intellectuel en Afrique(II). Un détour historique à la période d'avant Ahidjo s'avère nécessaire.

\section{BREF HISTORIQUE DE L'AVANT AHIDJO}

Les débuts du Cameroun actuel sont étroitement liés aux rivalités qui opposent les puissances coloniales européennes: France, Grande Bretagne et Allemagne.

C'est en 1884, que le diplomate allemand Nachtigal établit le Protectorat du Kamerun allemand (possession reconnue par l'Europe à la Conférence de Berlin , en 1885) qui durera jusqu'à la fin de la 1ère guerre mondiale. Les allemands ont laissé derrière eux un pays pacifié, avec des peuples stabilisés sur leur territoire respectif. Les réalisations allemandes sont impressionnantes ${ }^{16}$ : construction des premières routes (kribi-Yaoundé), le chemin de fer de l'Ouest et celui du Centre..., aménagement d'un ensemble de plantations dans le Sud, etc. L'enseignement est laissé aux missions, et les allemands ouvrirent leur pays aux étudiants camerounais.

A la fin de la Première Guerre mondiale, deux tranches orientale et méridionale sont découpées pour être rattachées à l'A.E.F. (Afrique Equatoriale Française), cependant que le reste du Cameroun est partagé en deux mandats internationaux: 4/5ème du teritoire dévolus à la France et une étroite frange ouest, revenant à la Grande Bretagne.

Les deux nations européennes allaient désormais chacune imprimer sa marque, introduire son organisation politique et économique, son système de pensée, sa langue et sa religion. En zone française, le pouvoir centralisé est monopolisé par la métropole. Sous le système de l'assimilation propre aux Français, les populations du Sud, peu structurées (acéphales) subissent profondément la pénétration culturelle, sociale et religieuse. La politique d'administration directe permet un essor économique important, profitant des antécédents allemands et 
utilisant toujours le recours du travail forcé. Le développement des cultures d'importation se poursuit ainsi que celui des infrastructures (port de Douala, aéroport...). Au Nord Cameroun (Ahidjo en est originaire), cette politique se heurte à la résistance des sociétés hiérarchisées et rebelles à l'occidentalisation. Les chefferies de l'Ouest Cameroun se révèlent également difficilement pénétrables.

Les Britaniques quant à eux, administrent leur mandat par leur système de "l'Indirect-rule" dans lequel les camerounais sont d'avantage associés à l'administration -comme une adjonction au gouvernement colonial du Nigeria, le pays voisin.

Durant la Seconde Guerre mondiale -la propagnade allemande parlant de récupérér le pays- le Cameroun se rallie très vite à la France libre, grâce aux efforts du Gouverneur Eboué.

Les orientations des administrations françaises et britaniques commencent à changer après 1946, lorsque le Cameroun occidental et oriental passent sous tutelle des Nations-Unies. Le Cameroun oriental est englobé dans l'Union Française en tant que " territoire associé ". Les populations des deux territoires commencent à anticiper l'autonomie gouvernementale et de nombreux groupes politiques se forment en même temps que les aspirations à l'indépendance sont énoncées.

Après 1948, l'Union des Populations du Cameroun (U.P.C) - Section locale du Rassemblement Démocratique Africain (R.D.A.)- revendique la réunification des deux territoires et l'indépendance, par rapport au gouvernement français, sous la conduite de Ruben Um Nyobè, qui jouit du soutien des milieux progressistes européens.

Les activités anti-françaises s'intensifient durant les années 50 , tandis que l'influence de l'U.P.C. s'accroît. Ce parti se retrouve dans la clandestinité. La mort de Um Nyobè et la chasse aux leaders historiques du mouvement, finissent par inhiber le dynamisme de ce parti.

L'évolution institutionnelle s'accélère avec le statut d'association et la formation du premier gouvernement, en 1957, par André Marie Mbida, Ce dernier sera rapidement démis par les français et remplacé en février 1958 par Ahmadou Ahidjo, musulman du Nord et ancien ministre de l'intérieur qui proclame l'indépendance le 1er janvier $1960 . .$. 


\section{LA TRAPPE ET LA TRAQUE DES INTELLECTUELS}

Nous savons grâce aux travaux de J.-F. Bayart que l'Etat au Cameroun s'est constitué et consolidé à partir de l'absorption progressive -par la classe politique dominante- de toutes les "forces sociales contre-hégémoniques": les syndicats, les partis politiques (mouvements nationalistes à l'instar de l'UPC (Union des populations du Cameroun), Partis politiques anglophones et francophones), les oppositions politiques internes et externes, les autorités traditionnelles, les grandes forces associatives ethniques telle que le "Ngondo" chez les Duala, les Eglises (Eglises catholiques, notamment), l'armée, et surtout les intellectuels ${ }^{17}$.

L'absorption s'est réalisée par la ruse, mais surtout par la coercition et la terreur au moyen d'une politique centralisée et fortement centralisatrice dans tous les domaines, de l'avènement du parti unique ("unifié"), la prééminence de l'administration publique. Bref, par ce que nous appelons "un quadrillage politique et administratif des populations" ${ }^{18}$.

Le projet hégémonique de Président Ahmadou Ahidjo, fondé sur l'éthique/mystique de l'unité nationale s'est révélé être un projet de domination de l'ensemble des segments de la société; un contrôle social. Aussi en 1977, Ahidjo déclare-t-il aisément, parlant de son projet de société, que ce dernier "est l'expression de [sa] vision du Cameroun de demain", "un projet global qui vise tous les domaines de la vie nationale"19.

Dès 1962, certains leaders politiques s'opposent à l'intention d'Ahidjo de former "un parti unique". L'idée faisait son chemin dans de nombreux pays africains; idée qui inspirait à tous de faire bloc autour d'un seul parti et d'un seul homme. A partir de ce moment, Ahidjo adoptera une stratégie qui sera d'ailleurs constante. Il utilisera d'un côté la persuasion et la ruse pour la recherche de ralliements individuels à son parti, à sa politique et à sa personne, et de l'autre côté, il n'hésitera pas à utiliser la répression contre toute opposition à ses objectifs politiques. La carotte et le bâton permettront ainsi au chef de l'Etat camerounais de réaliser -après une lutte contre la rébellion et les opposants politiques- un "parti unique": UNC (Union nationale Camerounaise) en 1966, et six ans plus tard, la République Unie du Cameroun.

Comme nous tenterons de l'illustrer "la fondation de l'UNC a eu pour conséquence "d'affirmer le contrôle du régime sur les intellectuels" ${ }^{20}$. En effet, le Parti-Etat a contribué au développement de l'Etat prébendier, à la fonctionnarisation des 
intellectuels. Pour asseoir sa suprématie sur les différents segments de la société, le sytème de parti unique, par différentes techniques, a violemment combattu toutes les vélléités d'opposition au pouvoir en place. Ceci revenait donc pour les intellectuels à se poser cette interrogation shakespearienne: "To be or not to be "(Etre ou ne pas être), que nous traduisons ici -en nous servant d'expressions camerounaises: "être quelqu'un", entendez jouir des avantages et des privilèges du pouvoir, ou "ne pas être quelqu'un", ce qui signifie le contraire. "Etre" se traduit par le ralliement/collaboration et le "ne pas être" par l'opposition/la contestation, ou plus exactement, "l'interrogation", "le questionnement permanent qui maintient l'esprit en alerte et nous fait découvrir à chaque étape nos limites et l'ampleur de notre ignorance " ${ }^{21}$.

\section{Ralliements à l'Etat prébendier.}

Des entretiens réalisées en janvier 1993 avec d'anciens étudiants de Paris dans les années 60-70 confirment les actions et les appels du gouvernement en vue du ralliement des étudiants à la politique de l'UNC. Nombreux reconnaîssent leurs sympathies à l'époque pour l'UPC, le principal parti d'opposition engagé dans la lutte révolutionnaire. Ils étaient affiliés auprès de l'UNEK (Union nationale des étudiants du Kamerun) "marxisante", très active dans les campus universitaires parisiens et européens, d'où ils critiquaient sévèrement Ahidjo et son régime. Pour bon nombre d'entre eux, le régime en place au Cameroun était composé de personnes "intellectuellement" incompétentes, ce qui justifiait leur incapacité à tenir tête au "néocolonialisme", à batir un Etat national/nationaliste, et surtout à dialoguer. Cette incapacité "intellectuelle" expliquait en grande partie la limitation des libertés d'expression, de la presse, de l'information, et le recours à la répression pour mettre au pas "les rebelles" et les "terroristes".

Par sa vocation d'éducateur, d'éveilleur de conscience, l'intellectuel est une denrée rare qui en se ralliant solennellement au régime au pouvoir lui apporte une légitimité et un crédit supplémentaire.

Les ralliements des intellectuels étaient donc recherchés. Plusieurs méthodes étaient utilisées: -en plus de la violence (brimade, délation, humiliation, similacre d'assassinat, etc...)- les appels aux ralliements (par voie de discours ou par l'envoi d'émissaires à l'étranger), des offres de privilèges, les promotions accélérées 
dans les structures politiques et administratives, et le contrôle sur l'Université, à travers l'intégration des intellectuels (universitaires) dans le système.

\section{$\underline{\mathrm{La}}$ fonctionnarisation des intellectuels}

Comme Houphouët Boigny en Côte d'Ivoire, dès le début des années 60, "les agents de récupération d'A. Ahidjo parcourent l'Europe où séjournent un nombre inconsidérable d'intellectuels camerounais allergiques à sa politique, développant sans relâches la thèse cynique des bonnes places qui n'attendront pas toujours "22. Les structures créées (Fonction publique, école d'administration et écoles spécialisées) à cet effet assurent une intégration rapide la plupart du temps sur la base du diplôme présenté. Les salaires et frais de mission sont importants, ainsi que les avantages liés à la fonction. Comme nous dit par exemple ce fils de haut fonctionnaire: "Nous avions près de sept hommes et femmes de maison, trois chauffeurs et des prisonniers mis à disposition pour des travaux manuels ...". D'autres avantages en nature: voitures de service, aliments, la pension et la retraite garantie, surtout des nominations et promotions rapides étaient reservés aux plus fidèles des ralliés.

\section{Le culte de la nomination}

Au Cameroun existe le culte de la nomination. Attribut constitutionnel, la nomination est une arme que possède le chef de l'Etat pour récompenser ceux des agents les plus "dociles" ou les plus zelés, c'est-à-dire qui suivent et défendent aveuglément la ligne politique du gouvernement. La nomination est perçue comme "une manne qui tombe du ciel", un manière de récompenser la chèvre qui a longtemps erré et qui se trouve désormais liée à son poteau: "la chèvre broute la où elle est attachée" pour faire allusion à "la politique du ventre", telle que décrite par J.-F. Bayart dans 1'Etat en Afrique.

La "camerounisation des cadres" se traduit par une "éclosion de postes administratifs"; les années 60-65 sont "l'époque de l'éclosion quasi éruptive des ministres, directeurs et chefs de Cabinets, ambassadeurs, colonels, préfets et sous-préfets, directeurs d'office de toutes sortes, présidents-directeurs généraux de régies ou d'entreprises nationales. Quiconque n'est pas encore quelque chose [nous disons "quelqu'un"] se croit appelé à le devenir bientôt. C'est un feu d'artifice ininterrompu de nomination, d'avancements, de promotion, de parachutages; il n'en fallait pas davantage pour faire perdre la tête à une bonne partie sinon 
à la majorité des fonctionnaires déjà en place et des diplômés d'université alors peu nombreux. C'est un cyclone épouvantable qui va dévaster la société camerounaise jusque vers 1965 [avant le parti unique], déchaînant prévarications et courses au plaisir, vices et jalousies"23.

Cette présentation n'est pas excessive. Il suffit, pour le comprendre, d'écouter les gens vous parler de leur nomination, de leur attente du journal parlé de 13 heures à la radio (la télévision n'existait pas encore à l'époque), ou de lire les très longues listes des récipiendaires dans le quotidien officiel Cameroon Tribune. L'Etat prébendier est en ce moment là en marche et, aiguise même les appétits des "incorruptibles". La nomination tombe alors du ciel et, est interprétée comme une "grâce" du chef de l'Etat: la délégation d'une parcelle de ses pouvoirs, une récompense des bonnes brebis. Des études démontrent assez clairement que la "camerounisation des cadres" a d'abord contribué à accroître de manière exponentielle les effectifs de la fonction publique ${ }^{24}$.

Il est d'ailleurs intéressant de noter que dans la stratégie de fonctionnarisation des intellectuels, le droit administratif camerounais reconnaît à l'époque à l'Université de Yaoundé une personnalité juridique autonome (autonomie de gestion et financière, mais non budgétaire); mais qu'il est dirigé par un Chancelier "délégué du gouvernement auprès de l'Université", qui possède des pouvoirs exhorbitants sur le conseil d'administration et sur les sections permanentes dudit consei ${ }^{25}$. Il s'agit ni plus ni moins d'un moyen de subordination et de contrôle de l'université afin d'obtenir des intellectuels souhaitant y faire carrière leur adhésion à la politique d'Ahidjo $^{26}$.

Adhésion des intellectuels à la politique d'Ahidjo.

Ralliements spectaculaires et nominations symboliques des intellectuels

L'une des premières personnalités intellectuelles camerounaises francophones à s'être ralliée spectaculairement au régime Ahidjo est sans doute William Aurelien Eteki Nboumoua en août 1961 au poste de Ministre de l'Education nationale.

En effet, dès 1960, face à la sympathie des étudiants camerounais de Paris (Centre culturel camerounais et UNEK), Ahidjo joue la carte de l'ouverture et agite les 
possibilités de carrière dans la fonction publique et l'accession rapide aux plus hauts postes de responsabilité.

D'autres ralliés éminents occuperont d'importantes fonctions et surtout, ministérielles: citons, parmi d'autres, François Sengat Kuoh, Victor Kamga, Vroumsia Tchinaye, Philémon Beb à Dong, Paul Biya (actuel président de la République), Simon-Pierre Tchoungui, Adamou Ndam Njoya, l'écrivain Ferdinand Oyono.

La plupart de ces personnalités ont la particularité et l'avantage de venir de différentes régions et d'être présentées comme la jeune classe montante dont Ahidjo compte se servir pour s'opposer et évincer les anciens "caciques" de la politique camerounaise (l'ancien Premier ministre André Mbida et les leaders des partis de l'opposition Mayi Matip, Charles Okala, Bebey Eyidi) que Ahidjo n'hésitera pas à qualifier de "quarteron d'hommes politiques dépassés" après que ces derniers se soient opposés -au travers d'un manifeste- à sa volonté de constituer un "parti unifié" [unique].

Ainsi par exemple, Beb à Dong originaire du département du Mbam est un "remplaçant" de Charles Okala. Il en est de même pour Paul Biya originaire du Sud peut contrecarrer Charles Assale, Simon Pierre Tchoungui (futur Premier ministre) d'origine beti, pour remplacer André Mbiba ancien Premier ministre, Eteki Mboumoua et Senghat Kuoh à la place des Soppo Priso, Bebey Eyidi ou Abel Kingué, ces deux derniers étant des leaders de l'UPC.

Les intellectuels camerounais francophones ne sont pas les seuls à participer au projet hégémonique du président Ahidjo. Après la réunification des deux Cameroun en 1961, on note l'entrée des intellectuels anglophones dans les gouvernements fédéraux. Notons: Endeley, John Ngu Foncha, Azikiwé, Nzo Ekhah Nghaky, Emmanuel Egbé Tabi, Salomon Tandeng Muna, Bernard N. Fonlon, Tatah Sakah, Dorothy Njeuma, Monie NKengong, Mfor Gwei.

Le ralliement des intellectuels au pouvoir d'Ahidjo se transforme en "sainte alliance" vers les années 1974-1975. 
$\underline{\text { La Sainte alliance entre les intellectuels et Ahidjo }}$

L'agitation universitaire commencée en 1969, (affaire des tracts) deux ans après l'ouverture de l'université fédérale, atteindra son comble en 1972-1973 avec les affrontements entre les étudiants et les forces de l'ordre. A l'origine de cette agitation, l'exigence du paiement des bourses d'étude constituait une des revendications des étudiants. "L'ordre règnera à l'université par tous les moyens ..." avait déclaré le Président dans un discours à la nation.

L'une des armes d'Ahidjo sera alors d'associer de plus en plus d'universitaires au projet hégémonique et d'assurer le contrôle de l'université (lieu sensible) par la nomination des intellectuels ralliés à la tête des instances universitaires.

Cette tactique aura des effets positifs pour le président Ahidjo, puisqu'en décembre 1974, à la suite du CESRST (Conseil de l'enseignement supérieur et de la recherche scientifique et technique), le Conseil des affaires culturelles et la FENAC (Fédération des étudiants camerounais - protestante, proche de l'UNC) adresseront des motions de soutien au chef de l'Etat à la veille du "Congrès de la maturité" à Douala. Le CESRST recommandera solennellement à l'issue de la réunion de décembre 1974 que l'université s'engage désormais plus politiquement en se conformant aux principes politiques d'unité et de paix nationales dégagés par l'UNC.

L'université participera désormais officiellement à la politique générale du parti. Des intellectuels -pour la plupart des hommes de sciences ${ }^{27}$ - se retrouvent en nombre très important à la présidence de la République (Secrétariat du gouvernement), dans les gouvernements, dans les organes centraux du parti, à la tête des établissements universitaires et des grandes écoles, au Conseil économique et social et dans les hautes administrations.

A titre d'exemple, M. Guillaume Bwélé (agregé de lettres) qui deviendra ministre de l'Information et de la Culture en 1979, est d'abord passé par la présidence de la République comme conseiller technique puis conseiller spécial auprès de Ahidjo. De même l'anglophone Bernard Fonlon devient ministre adjoint des Affaires étrangères en 1964 après avoir été chargé de mission à la présidence de la République. De nouveaux intellectuels font progressivement leur entrée dans le gouvernement: Njensi (1970), Christian Songwé Bongwa; Joseph Awunti Chongwain (tous diplômés en Angleterre)(1972), Dorothy Njeuma;

Monie Nkengong, Amadou Ndam Njoya, Marcel Yondo et René Ze Nguelé (1975), Salomon Mfor Gwé et Guillaume Bwélé (1979). 
Certains intellectuels cumulent plusieurs postes de pouvoir au sein des instances universitaires, mais également ailleurs. On peut citer les cas de Bol Alima, Directeur de l'ENSA (Ecole nationale supérieure agronomique (1975-1979), directeur général du Centre universitaire de Dschang dès $1978^{28}$, membre du Conseil économique et social depuis 1975 et du Comité central de l'UNC (parti unique) à partir de 1980.

Il est donc clair que les intellectuels camerounais francophones comme anglophones, dans des proportions sans doute différentes, ont participé de manière consciente (collaboration) ou inconsciente (de bonne foi) au projet hégémonique du président Ahidjo. Ils ont participé à la définition des fondements intellectuels et théoriques de ce projet et à justifier les actes et la politique du régime.

P.-F. Ngayap dans son étude sur la place de l'intelligentsia dans le personnel politico-administratif du Cameroun ${ }^{29}$, note que sur l'ensemble des 131 membres des gouvernements Ahidjo depuis 1958: 65\% ont fait des études supérieures, dont $23 \%$ possèdent un diplôme supérieur à la licence (baccalauréat +3 ans): doctorats français ou à la française, agrégations, doctorats anglo-saxons, DES (diplômes d'études supérieures), diplômes d'ingénieur, Master's, etc ... et $42 \%$ ont fait leurs études jusqu'à la licence ou à un niveau équivalent ( 3 années d'études après le baccalauréat); $35 \%$ ont un niveau d'études secondaires et primaires dont $20 \%$ ont un niveau d'études secondaires et $15 \%$ ont le niveau primaire.

L'auteur note également que le niveau général d'études des membres du gouvernement s'est élevé au fil des remaniements ministériels: L'équipe fédérale du 12 juin 1970 est composée de 20 membres parmi lesquels 17 ont un niveau supérieur ou égal à la licence ( 9 ont le niveau de licence et 18 un niveau supérieur à la licence); celle du 30 juin 1975 compte 29 membres, dont $90 \%$ possèdent un diplôme de l'enseignement supérieur ( 9 niveau de licence et 17 un niveau supérieur à la licence). Le gouvernement de juillet 1980 fort de 29 membres a un pourcentage des diplômés de l'enseignement supérieur sensiblement identique au gouvernement précédent.

Bien que le nombre des diplômés de l'enseignement supérieur soit croîssant, il est tout à fait juste de reconnaître que l'accrôissement seul du nombre des intellectuels ne compte pas valablement pour la prise de décisions ou pour la bonne gestion des affaires publiques. Ce qui compte le plus c'est sans doute la qualité de ces intellectuels.

Ceux qui ne collaboraient pas au régime étaient considérés comme des "terroristes" ou des "subversifs" -aujourd'hui, on les qualifie d " opposants ". Parmi eux, 
de nombreux intellectuels. Le régime Ahidjo ne ménageait aucun effort pour les pourchasser, les traquer et les contraindre au ralliement ou à l'exil.

\section{La chasse aux maquisards et aux subversifs}

Le régime Ahidjo avait plusieurs techniques ou tactiques pour contraindre les intellectuels au ralliement. Tous ceux qui refusaient les offres de l'Etat prébendier s'exposaient à subir l'intimidation ou la persécution, la répression, la torture (simulacre d'assassinat), et surtout, l'élimination physique.

\section{Intimidation et persécution}

L'objectif est double: préparer les conditions psychologiques du ralliement et/ou obtenir un "ralliement négocié", c'est-à-dire qu'un marchandage se fait entre les autorités spécialisées dans ces actions psychologiques, et l'intellectuel concerné. Ce dernier acceptant d'échanger son silence contre l'autorisation que lui accorde de régime de mener ses activités, ou à travailler en toute liberté et quiétude.

Les exemples sont nombreux. Tout commence par le "chemin de croix" que doit parcourir tout diplômé postulant à un emploi dans la fonction publique ou à l'université. Le système de recrutement est infantilisant. Il faut à chaque niveau du circuit (service et direction chargés du recrutement) faire preuve de son adhésion "indéfectible" à la logique et au fonctionnement du système et de son "indéfectible attachement" à la personne du chef de l'Etat (tenue à l'éffigie du chef de l'Etat ou aux couleurs du parti, carte du parti unique, etc...). Le dramaturge camerounais Raymond Ekossono traite de cette question dans la pièce de théâtre Le soleil rouge

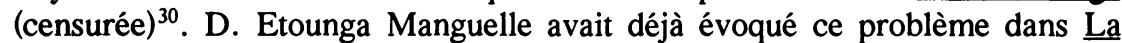
colline du fromager où il présente la suspicion et la méfiance qui pèsent sur les étudiants camerounais ayant séjourné à l'étranger en France plus particulièrement: "Moussaka devenu chargé de mission à la Présidence de la République reçoit $N$ 'Tam, un ancien camarade de classe, de retour de France et à la recherche de travail, et dont les demandes n'ont jamais de suite alors qu'il est hautement qualifié; il lui explique que le régime est en train de se mettre en place, et pour durer il a besoin de choisir des hommes de confiance, donc de se méfier des jeunes qui ont vécu longtemps en France, aux idées inadaptées au pays, qui parlent sans cesse de démocratie, de liberté de presse, de multipartisme, etc" (p. 87). De plus, 
si l'étudiant a la "chance" d'être recruté, une période probatoire de six mois sans solde permet de jauger de sa docilité et de sa capacité à intégrer le système sans rechigner.

J.-F. Bayart dans L'Etat au Cameroun, cite l'exemple de l'upéciste Abel Eyinga et ses amis, qui, fidèles à leur opposition, avaient dû négocier en 1961 avec les autorités du régime en place afin de continuer d'animer le Cercle culturel camerounais et de publier la Revue camerounaise. Il fallut attendre l'entrevue avec Ahidjo le 24 février 1961 pour que soit scellée "une collaboration sans arrières pensées avec les responsables camerounais" ${ }^{\prime 31}$.

On peut également évoquer l'exemple cité par Abel Eyinga dans une réplique au journaliste du Monde $P$. Biarnès ${ }^{32}$ au sujet de l'arrestation arbitraire dont le polytechnicien Jean-Jacques Ekindi avait été l'objet en 1970 alors qu'il venait en vacances au Cameroun. Arrêté pour "subversion" (sic) par le SEDOC (Services des études et de la documentation, services des renseignements) et traduit devant le tribunal militaire de Yaoundé après deux mois de détention. On lui reprochait "d'avoir écrit étant à Paris, des articles critiques contre le gouvernement de $M$. Ahidjo dans le bulletin de l'Union nationale des étudiants camerounais (sic)".

Des entretiens réalisés auprès d'anciens étudiants camerounais des années 70 révèlent de nombreux cas de ce type. Certains étudiants racontent comment il leur était arrivé de trouver leur appartement saccagé, les matelas éventrés, tout sens dessus dessous. Ils attribuent ces actes à la police secrète du régime qui infiltrait leurs associations étudiantes. Le "calvaire" vécu par Mongo Beti pendant la saisie de son ouvrage Main basse sur le Cameroun: autopsie d'une décolonisation est révélateur des mécanismes et des pratiques d'intimidation du régime qui n'hésite pas à se faire aider et à étendre son réseau hors du Cameroun (pp. 16 et s.).

\section{La répression légale}

La répression au Cameroun est avant tout légale. Elle s'appuie sur un arsenal juridique bien ficelé. Ce qui permet à Ahidjo de déclarer aisément au cours d'une conférence de presse le 2 juillet 1963 (en substance): "Je sais que ces Camerounais, qui soutiennent la subversion indirectement par des attitudes équivoques, comptent sur le fait que la Justice, si on les appréhendait, en l'absence de preuves matérielles, les relâcherait. Mais nous disposons d'armes légales qui feront en sorte que, même si la justice les relâchait, si nous avons la conviction que, d'une façon directe ou indirecte, ils aident les terroristes, nous les enfermerons". La législation 
d'exception (état d'urgence) décrétée par l'Ordonnance de mars 1962 peut à tout moment être invoquée pour justifier tout acte de répression.

C'est sur la base de ce texte que l'on censure les informations, les journaux, la littérature et les ouvrages jugés "subversifs". C'est aussi à ce texte que l'on recourt pour justifier la détention administrative d'un individu pendant de nombreux mois sans procès. Ce texte a trouvé ses premières applications avec l'arrestation et la disgrâce politique des intellectuels leaders de partis d'opposition qui avaient osé au travers d'un manifeste de juillet 1962- exprimé leur désaccord sur la volonté d'Ahidjo de créer un "parti unifiée" (unique). Un complot leur fut imputé et permit leur arrestation et leur emprisonnement après un procès-éclair.

L'arsenal juridique est renforcé par des appareils répressifs, tels que la Direction des affaires politiques du Ministère de l'Administration territoriale et le ministère de l'Information et de la Culture pour tout ce qui concerne la censure. Le SEDOC devenu DIREDOC (Direction des études et de la documentation), la BMM (Brigade mobile Mixte), (police spéciale) se chargent des pratiques de répression et de tortures. Il faut y ajouter les tribunaux et les camps de tortures militaires appelés "centres de rééducation civique" par le gouvernement. Les pratiques de ces organes ne sont pas très éloignées de celles de la Gestapo: ouverture des correspondances, persécution nocturnes non motivées, arrestation sans mandat d'arrêt souvent au milieu de la nuit, délation institutionnalisée, représailles contre les familles, etc ... Un français ayant vécu 43 ans au Cameroun livre un témoignage terrifiant de ce qu'il appelle la "Gestapo africaine" ${ }^{33}$.

Parmi les armes légales du régime, les procès sont également un moyen médiatique (surtout de désinformation) et psychologique en direction des opposants. Outre le procès de Victor Kamga, ancien Président de l'UNEK devenu ministre des finances d'Ahidjo (affaires des tracts de 1966) dans lesquels l'accusé tentait d'impliquer le chef de l'Etat dans des scandales financiers, l'affaire Ouandié/Ndongmo est selon nous le procès des intellectuels.

\section{Le procès Ouandié/Ngongmo: un procès contre les intellectuels}

On a souvent dit que l'action de Mgr Ndongmo et de son diocèse allait à l'encontre des intérêts économiques du néocolonialisme. Ceci est en partie vrai. Mais, il ne faut pas oublier que Mgr Ndongmo était perçu comme un prélat gênant dans la mesure où il avait une conception trop "intellectuelle" de sa mission, conception assez proche de la théologie de la libération développée en Amérique latine. Pour lui en effet, "L'Etat croit que nous devons prêcher un christianisme désincarné, 
parler du ciel, des anges, sans toucher aux réalités vitales de chaque jour. Or, l'Evangile du Christ n'est pas une théorie, mais une vie. Il s'insère dans toute la vie de l'homme engagé dans la famille, la politique, la profession et le syndicat" ${ }^{34}(17$. Cette conviction pousse l'évêque à impliquer son diocèse dans des entreprises économiques à finalité sociale. De plus, il avait un esprit indépendant, jouissait d'une bonne réputation auprès de la jeunesse et animait le journal $L$ 'Essor des jeunes -plusieurs fois censuré.

De l'autre côté, Ernest Ouandié, ancien Vice président de l'UPC -revenu au pays en 1962 pour prendre la direction du maquis, après 4 ans d'exil- était considéré comme le dernier "cerveau", le "dernier chef" de la rébellion locale, la "tête pensante" de la révolution. Déjà en 1957, il avait ouvert au Caire, avec la collaboration de deux autres leaders de l'UPC, Félix Moumié et Abel Kingué, une agence d'information d'où ils émettaient et diffusaient les émissions radiophoniques "La voix du Cameroun".

Le procès Ouandié/Mgr Ndongmo venait surtout après les agitations estudiantines de janvier-février 1969 et après le crime de lèse-majesté commis par le juriste Abel Eyinga qui, de Paris, posa sa candidature à l'élection présidentielle de mars 1970, contraignant Ahidjo à mener campagne contre un candidat "fantôme". Ce dernier fut jugé par contumace et condamné à huis clos par le tribunal militaire à 5 ans de prison. Ernest Ouandié est capturé le 7 juin et Mgr Ndongmo le 28 août 1970 après que l'Agence France Presse ait révélé la découverte d'un stock d'armes dans l'usine Moungo-Plastique financée par le diocèse de l'évêque (!).

Le procès Ouandié/Ndongmo était le procès de ceux qui sont trop "éclairés"; "ceux qui voient trop clair" pour reprendre une expression camerounaise. D'autres intellectuels ont été purement et simplement liquidés.

\section{Liquidations physiques et assasinats politiques}

Les liquidations symboliques majeures sont celles des leaders intellectuels de l'UPC, principal parti d'opposition ayant été poussés dans l'illégalité (actions armées dans le maquis). On citera le cas de Ruben Um Nyobé, le "Mpodol", le sauveur en langue bassa, qui aurait été tué en septembre 1958, dans la forêt bassa, à en croire le journaliste français Georges Chaffard, "parce qu'en s'enfuyant il emportait à la main une serviette, on l'avait pris pour un "intellectuel" (sic), et malgré les ordres, on avait tirén ${ }^{\prime \prime 35}$. Cette version de la mort de Um Nyobé est ahurissante ${ }^{36}$. Mais, on peut tout simplement noter que c'est bien parce qu' "on l'avait pris pour un intellectuel" qu'on avait tiré sur lui. Pourquoi cet auteur précise 
t-il que Um Nyobè avait été pris pour un intellectuel $?^{37}$ Peut-on pour autant dire les intellectuels, et plus particulièrement ceux qui dirigeaient l'UPC, étaient la cible des soldats "loyalistes" et du régime Ahidjo. La réalité confirme cette hypothèse.

En effet, depuis l'interdiction du droit de grève en 1948, les dockers de Douala (les travailleurs) en général ne pouvaient plus manifester ouvertement leurs sympathies pour l'UPC, il restait les intellectuels qui étaient pour la plupart suspectés de sympathies révolutionnaires.

On sait aussi que Félix-Roland Moumié sera empoisonné à Genève en Suisse par un soit-disant journaliste. En réalité, il s'agissait d'un agent des services secrets français, nommé William Bechtel arrêté en Belgique en 1974 et extradé en Suisse, où il n'a jamais pu être jugé!.

On ne saurait également oublier de citer le cas du célèbre économiste Osendé Afana, assassiné dans le front Sud de la rébellion ${ }^{38}$. A en croire G. Chaffard, Ahidjo a toujours espéré le ralliement de cet intellectuel de renom. Ahidjo aurait ordonner de le ramener vivant à Yaoundé. Et il est vrai que ce dernier aurait été "une recrue de qualité, dont l'amende honorable aurait [eu] du retentissement chez les étudiants et les intellectuels encore en état de rébellion morale ..." (t. II; p. 346).

La trappe et la traque des intellectuels ont souvent été des actions psychologiques censées annihiler toute velléité d'opposition à la politique d'Ahidjo. Elles peuvent être interpretées comme une lutte contre l'UPC, entendu comme "parti des intellectuels" pour les raisons déjà évoquées plus haut.

Le régime Ahidjo s'est ainsi progressivement consolidé à partir d'une collusion entre élites politiques et élites universitaires. Le régime de terreur et de peur instauré, et la chasse ouverte aux autorités camerounaises pour/contre les intellectuels ont poussé ces derniers à deux attitudes: le mutisme et/ou la "collaboration" dans le plein sens du terme, ou le contraire l'exil intérieur -dans une prison (la mort pour les plus intransigeants)- ou extérieur (à l'étranger): "être ou ne pas être".

Toutes ces actions ne tuent pas et ne taisent pas les contestations estudiantines (nouvelle affaire des tracts en 1976), les opppositions symboliques comme celle d'Abel Eyinga, candidat à la Présidence de la République. Au contraire, elles font naître et développer toute une littérature originale et sans complaisance, 
qualifiée à Yaoundé de "subversive", et donc censurée par voie de conséquence. On assiste alors à l'émergence -à côté du véritable maquis (la lutte armée)- d'un "maquis intellectuel".

\section{LE MAQUIS "INTELLECTUEL"}

Nous avons vu que le régime Ahidjo interdit toute opposition politique à l'intérieur du pays: le procès Ndongmo/Ouandié en est la preuve. La plupart des opposants au régime manifestent et se manifestent de l'étranger. Cette forme de contestation et d'opposition ne semble pas efficace, parce qu'elle mal organisée et " encadrée " par les lois et les pratiques politiques des pays d'accueil, en Europe en particulier, elle ne se fait pas intense, pesante et régulière.

Nombreux sont ces intellectuels qui muselés, font appel à la muse. Le débat politique quitte les arènes officielles pour se retrouver dans des écrits, dans la littérature.

Il faut reconnaitre avec J. Ackad $^{39}$ - parlant des écrivains francophones - que la plupart des romanciers camerounais sont des "intellectuels" (sic) qui ont fréquenté les écoles missionnaires et les lycées de leur ville avant d'aller poursuivre en France des études universitaires où la plupart du temps ils restent en exil. Dans leurs écrits, les trames retracent assez souvent, de manière extrêmement douloureuse, l'expérience dictatoriale vécue dans le pays, l'incurie et la corruption des fonctionnaires et agents des dirigeants locaux, etc ... Ils tentent d'ailleurs d'apporter des solutions alternatives aux problèmes du pays.

\section{Critique de l'Etat et de la société tous azimuts}

Comme note, à juste titre, J. Ackad dans son étude sur le roman camerounais, les premiers écrits des auteurs littéraires camerounais (1950-1960) sont anticoloniaux et anticléricaux. Ils usent "abondamment de l'ironie ${ }^{40}$ pours 'attaquer aux aspects les plus manifestes de l'occupation et de la domination étrangère dont le pays 
fut victime pendant longtemps" (p. 8). Après l'indépendance, commence une période de critique sociale: on critique surtout des problèmes socio-culturels relatifs à la société indépendante (dot, polygamie ...).

Il faut attendre le début des années 70 , notamment après l'affaire Ndongmo/Ouandié et la condamnation à mort de ce dernier leader du seul parti d'opposition réellement connu l'UPC. On verra donc, de nombreux auteurs comme Mongo Beti, sortir de leur exil intérieur et publier une série d'ouvrages "politiquement" engagés dont le célèbre et premier pamphlet politique du pays Main basse sur le Cameroun. En 1980, le " cri " vient de l'intérieur du pays, de la part de Jean Marc Ela ${ }^{41}$, est également un acte d'engagement et d'opposition ouverte, et une contribution majeure, à la démystification de l'Etat postcolonial et surtout de son administration. Deux ans plus tard, un autre auteur opposant également au régime Ahidjo, publie en 1982 sous le pseudonyme de Yodi Karone, un ouvrage intitulé Le Bal des Caïmans dans lequel l'auteur fait un procès virulent de l'injustice sous toutes ses formes, juridique principalement et de la répression dans un pays africain qui, bien qu'il ne soit pas explicitement nommé rappelle assez étrangement le Cameroun.

\section{Mongo Beti, l'écrivain engagé, le révolutionnaire, le briseur de tabous}

Main basse sur le Cameroun est selon nous un ouvrage en avance sur son temps puisqu'il correspond à une époque particulière de la politique africaine de la France. L'auteur y démonte les mécanismes de la sujétion des dirigeants locaux, la répression des populations et le soutien aveugle de la France au régime camerounais. Ainsi par exemple à propos de l'arrestation de Mgr Ndongmo pour participation à un complot contre le régime, Mongo Beti trouve que plusieurs personnes ont des intérêts dans ce procès: "le lobby d'outre-mer de Paris, sans doute le plus réactionnaire, le plus obtus de toute l'histoire des colonisations, y trouve une source de profits inouis, auxquels son esprit de routine et de fraude n'est pas prêt de renoncer. Les gouvernants camerounais n'ont jamais douté, quant à eux, que leur survie politique ne fût étroitement liée à ce mode d'exploitation de leur pays et de leurs "frères". Le régime gaulliste, pour sa part, outre l'intérêt économique de posséder, pour ses exportations, un exutoire de tout repos, a réussi à tourner le drame à son profit politique, en présentant à l'opinion cette lamentable situation comme une fatalité qui requiert précisément la "présence" et l'aide de la France sous la forme de la "coopération", pour être combattue avec quelque chance de succès" (pp. 130-131). 
Le régime Ahidjo n'est pas épargné. Traitant des rapports et échanges entre village et bidonville, l'auteur constate qu'il s'est étoffé sous Ahmadou Ahidjo, démontrant qu'au lieu de les intégrer la ville africaine issue de l'indépendance gaulliste rejette au contraire les pauvres, l'immense masse des Africains: "C'est que l'aristocratie bureaucratique, qu'on appelle à tort bourgeoisie africaine, tant cette classe manque d'assise économique, d'idéal collectifet d'esprit d'initiative qui sont caractéristiques de la bourgeoisie européenne, s'est enfermée, elle, dans le ghetto de la consommation et de la jouissance. Elle s'est révélée une classe aussi inutile que vorace, décevant tout le monde, aussi bien la masse des Africains qui l'imaginaient appelée à les guider dans la voie de la dignité (...) En perdant tout contact avec les masses elle laisse le régime pour ainsi dire en l'air, dans l'obligation d'interposer, à défaut d'une courroie de transmission entre le peuple et lui-même, la police et l'armée, avec toutes les conséquences qu'entraîne nécessairement cette sorte de dialogue" (p. 106).

Dans Remember Ruben paru en 1974 et La ruine presque cocasse d'un polichinelle (Remember Ruben II), ces romans mythiques brisent un tabou, puisqu'à travers le récit historique de la vie et du combat de Ruben, on revit la trajectoire du leader de l'UPC alors qu'il se battait pour l'indépendance intégrale de son pays. Nom tabou au Cameroun pendant une longue période, en énonçant et en évoquant le nom de Um Nyobé, Mongo Beti exhume un passé douloureux, escamotté dans les ouvrages d'histoire. Il présente ainsi une "épopée qui honore la mémoire de Ruben Um Nyobè, héros national et martyr du Cameroun". ${ }^{42}$

Or, nous savons bien que parler de Um Nyobè, c'est nier la paternité d'Ahidjo comme le fondateur du Cameroun moderne, cest lui renier le titre de "Père de la nation camerounaise", c'est du même coup considérer ce dernier comme un usurpateur. L'auteur commet donc là un crime de lèse-majesté qu'on qualifie juridiquement au Cameroun de "subversion", crime passible d'emprisonnement et souvent de la peine de mort comme nous le verrons plus loin dans l'ouvrage de Yodi Karone.

Dans Perpétue et l'habitude du malheur, Mongo Beti retrace les échecs de la lutte menée par le révolutionnaire Um Nyobè pour l'indépendance de son pays. Il relève surtout la situation plus que dramatique et désastreuse des hôpitaux publics qui est "pire qu'avant l'indépendance": "Perpétue ne serait sans doute pas morte si ce maudit pays n'était totalement dépourvu de médicaments depuis l'avènement de Baba Toura [...] un gouvernement noir fondant des usines avec l'argent des noirs, pour donner des médicaments à ses frères noirs, ça devrait être cela l'indépendance, non ?". Il convient de noter qu'il ne fait aucun doute que Baba Toura, "homme du Nord" (sic) est bien le président Ahidjo. En traitant des 
problèmes de femmes, des pauvres, des jeunes des villages qui sont marginalisés, Mongo Beti évoque la question des "cadets sociaux" décrite par J.-F. Bayart dans l'Etat au Cameroun.

\section{Yodi Karone et son "Nègre de paille"}

Yodi Karone dans le Bal des caïmans, poursuit comme Mongo Beti, la tentative de démystification du régime et de démystification du pouvoir d'Ahidjo et de l'homme providentiel. A travers l'histoire de "docta" (sic) Adrien ${ }^{43}$, un intellectuel, titulaire d'un doctorat en philosophie, opposant au régime en place et "militant subversif" (sic); de Père Jean, un curé illetré que des supérieurs ont disgrâcié parce que considéré comme fou, la trame de cette oeuvre rappelle étrangement la parodie de procès intentée à Mgr Ndongmo et le leader de l'UPC Ouandié. D'ailleurs durant la période du procès, Mgr Ndongmo était souvent traîté de fou, d'un intellectuel qui s'est trompé de vocation et de profession.

Yodi Karone poursuit plus tard sa diatribe contre le régime politique du Cameroun dans Nègre de paille un roman dans lequel il traite des libertés individuelles bafouées par le régime en place. Il semble y défendre l'idée affirmée plus tôt en 1979 par D. Etounga Manguelle: "Laisse nous parler (...) Maintenant nous sommes indépendants, chacun a obtenu le droit de dire librement ce qu'il pense" et par Benjamin Matip la même année dans Laissez-nous bâtir une Afrique debout.

\section{Bernard Nanga et Les chauves-souris}

Bernard Nanga dans Les chauve-souris critique l'instauration du parti unique et ses justifications; il dénonce le capitalisme outrancier, la gabégie d'un nouvel Etat, les abus de la classe dirigeante, les fonctionnaires véreux en mal de politique, jouisseurs invétérés, égoïstes, la délation ...

D'autres auteurs dressent un portrait ironique de la politique en Afrique.

\section{Regards sur la Politique.}

D. Ndachi Tagne dans son analyse critique des romans camerounais ${ }^{44}$, reconnaît que la politique en Afrique est avant tout une "stratégie pour vaincre son adversaire autant par la logique que par l'astuce". Mais, il semble bien que sur le continent africain, les pratiques politiques courantes sont largement et presqu'exclusivement 
teintées de machiavélisme et de mesquinerie. En effet, les agissements des hommes politiques au pouvoir, finissent par faire croire que la politique n'est que tromperie, manipulation, fourberie, corruption.

Ce vécu de la politique est assez bien rendu dans les écrits des romanciers camerounais avec beaucoup d'ironie et de dérision. Ainsi par exemple, F.-B. M. Evembe dans Sur la terre en passant ironise sur la carrière d'un ministre et ses collaborateurs. Il s'en prend au néocolonialisme et particulièrement à la "maffia des indispensables" que sont les collaborateurs blancs des ministres noirs. Bien que ne traitant pas spécifiquement du cas du Cameroun, le romancier camerounais Daniel Ewandé dans son titre à lui seul Vive le président! la fête africaine traite de manière comique mais ironique de la décolonisation en général et de l'Afrique mal partie en particulier. Il y distribue les points aux bons et mauvais présidents, il présente une image des ministres, des députés, des intellectuels, de la révolution, du capitalisme, de l'assistance technique ... et de 1'"Etalon-senghor", "intellectuel copieusement nourri de citations et de lieux communs".

Un autre auteur comme Medou Mvomo dans $A$ frika $B a$ 'a présente la politique à partir de l'exemple d'un maire qui, pour se faire réélire, est convaincu "qu'il [faut] amadouer les riches et construire des églises" alors qu'à "Nécroville" (ville de la mort) règne l'injustice, le chômage chronique, la pauvreté et la misère écrasante".

La politique est également synonyme de censure et surtout de répression. La mort de l'intellectuel Nzobé dans Afrika Ba'a témoigne bien, comme dit D. Ndachi Tagne, l'absence de libertés fondamentales dans les pays africains et justifie la méfiance vis-à-vis des questions politiques ${ }^{45}$. Décrite également par Yodi Karone, cette répression a souvent été une "chasse aux rouges" [communistes]: "voilà des années que tous les services de la police et de l'armée traquent sans relâche, à travers le pays, les derniers bastions de la révolte, fouillant villages et forêts, perquisitionnant à toute heure dans les logis, torturant pour un renseignement vital mais jamais confirmé, tuant passionnément le gibier insaisisable".

\section{Quelques idées alternatives}

Certains auteurs proposent des voies multiples pour sortir le pays des fléaux tels que l'injustice, la corruption, la démagogie, le gaspillage, la néocolonisation, l'acculturation, la pauvreté. D'autres pensent qu'il suffit tout simplement de changer 
les hommes: l'avènement des intellectuels au pouvoir permettra de combler le déficit intellectuel des dirigeants actuels.

\section{Les différentes philosophies}

L'étude idéologique du roman camerounais telle que réalisée par D. Ndachi Tagne permet de dégager quelques idées et conceptions du monde qui tiennent compte de l'histoire, des valeurs et des réalités camerounaises. On distingue:

- le "traditionalisme" ou le "mysticisme africain" de Charles Gabriel Mbock: Un pays comme le Cameroun ne peut se développer de manière harmonieuse en faisant l'impasse sur les souvenirs du passé, sur la sagesse et les valeurs ancestrales.

- L' "antitraditionalisme" de l'auteur anglophone A. Ngongwikuo: l'harmonie traditionel ayant été brisée par l'avènement du colon allemand, l'univers traditionnel doit tenir compte de l'ère nouvelle, celle d'une certaine liberté, du développement de la civilisation.

- le "pragmatisme communautaire" de Medou Mvomo: L'intellectuel a un rôle important à jouer. Il doit être pragmatique et contribuer à le reconstruction avec le souci des valeurs humaines.

- le "féminisme" de Zanga Tsogo (de son vrai nom Delphine Tsanga, ancien ministre des affaires sociales), de J. Owono et de Francis Bebey: L'importance de la femme ne doit pas être négligé.

- la "philosophie du néant" de F.-B. M. Evembé: Il faut faire raser le Cameroun postcolonial, un pays travesti et délabré, un pays sans valeurs fondamentales ni principes essentiels.

- le "populisme" du diplomate James Oto, pour qui la démocratie et les droits de l'homme doivent permettre l'épanouissement de l'individu dans une communauté libre et prospère.

- 1' "officialisme" de Pabe Mongo: l'idéologie officielle devrait assurer l'intercommunicabilité entre la tradition et le modernisme entre le pouvoir central et les populations.

- 1' "associatisme" de Francis Bebey qui est contre l'autoritarisme, le népotisme et la dictature d'un seul homme. Il est contre la personnalisation du pouvoir et croit en la force du groupe en action: syndicat, associations de femmes... Pour lui, "la voie du peuple est la voie de Dieu".

- l' "humanitarisme" d'Aoué Tchany: s'inspirant de la neutralité suisse, cette "idéologie "recherche le plein épounouissement de l'homme.

- Le "moralisme politique" de B. Nanga: Bien que ne proposant pas explicitement de solutions aux problèmes politiques soulevés, l'auteur oppose à l'individualisme un communautarisme traditionnel dans un régime où il ne règne pas la corruption, la tyrannie et le néocolonialisme. Il pense qu'une 
moralisation de la classe politique est nécessaire et qu'elle est appelée à tenir compte du paysannat et du sexe dit faible.

L'idée de moralisation de la vie politique sera reprise et amplifiée par Paul Biya après son accession à la présidence de la république.

- Le "nationalisme" d'I. Matiba: La lutte anticoloniale doit être poursuivie. Le colon est encore au pouvoir. L'indépendance reste à conquérir.

- "Nationalisme et le socialisme après la Révolution" (René Philombe, Yodi Karone et Mongo Beti):

Pour R. Philombe, le "peuple debout" doit se révolter et s'élever contre le néocolonialisme et contre le système mis en place par les occidentaux à travers des indépendances factices et l'érection des gouvernements fantoches. Yodi Karone fonde l'espoir du peuple dans la victoire de la révolution/lutte contre le système dictatorial.

Mongo Beti quant à lui, dans La ruine cocasse d'un polichinelle (Remember Ruben II) propose à travers le personnage de Ruben, que la résistance et la révolte doivent s'organiser contre le colon et le dictateur local. Il rêve de la construction d'une société collectiviste où les femmes jouent un éminent rôle. Fidèles aux idées du nationaliste Ruben Um Nyobè, Mongo Beti reste convaincu que le socialisme devra "inmanquablement" s'installer en Afrique pour le bonheur des masses déshéritées.

Une autre solution consiste également à changer les hommes et certains auteurs pensent que les intellectuels doivent exercer le pouvoir.

\section{L'avènement des intellectuels au pouvoir}

Certains auteurs comme D. Ewandé et F. Bebey se méfient des intellectuels. Le premier trouve que les intellectuels comme Senghor sont d'une abstraction et manque du sens de la communication, puisque par exemple "lorsque Senghor parle, chacun se rue sur son dictionnaire" (p. 100). F. Bebey dans La poupée ashanti ${ }^{(15)}$ souligne les dangers de la personnalisation du pouvoir et les risques de dictature qui guettent le "docteur" un des personnages du roman (en réalité: Kwamé Nkrumah) dont Princess se plaint de la publicité qu'on fait à la radio de l'action et du héros: "Pour moi, la radio c'est la musique qui m'intéresse, ce n'est pas du tout le reste (...). A chaque fois, on te dit que le docteur a fait quelque chose pour le pays (...) En fin de compte, je ne vois pas ce qu'il a fait pour le pays" (p. 68). 
D'autres auteurs comme Mongo Beti sont par contre convaincus que "C'est bien l'analphabétisme d'Ahmadou Ahidjo qui le pousse sur les premières marches de la vassalité et de la guerre civile" (Main basse sur le Cameroun 2 p. 73). J.-C. Aoué Tchany va plus loin que Mongo Beti. Pour lui, l'avènement des intellectuels au pouvoir est la solution qui permettra à l'Afrique de "sortir de la caverne(sic)" pour reprendre le mythe platonien. Partisan d'une "révolution anti-médiocrité", il propose la mise sur pied d'un régime technologique fondé sur une idéologie "humanitariste". Il faut une révolution aux trousses d'un Professeur (intellectuel): "Bientôt [dit Steve, un personnage de Aoué Tchany], le pays sera sans la botte des intellectuels. Nous changerons tout et le rêve de Platon sera enfin réalité" (p. 166).

Comme dit Karamba -un "intellectuel" de son état puisqu'il est titulaire d'un Brevet d'études du permier cycle (BEPC)- un personnage de Medou Mvomo dans Afrika $B a^{\prime} a$ il ne faut pas "(un) intellectuel contemplatif, [un] intellectuel négatif au sens où il se réfugie dans la démolition de l'acquis sans proposer et sans organiser la reconstruction" (p. 112). Il faut donc pour reprendre les idées d'Aoué Tchany un intellectuel pragmatique, pratique, réaliste, "humanitariste", il se préoccupe de "l'épanouissement de l'homme" (p. 35).

Mais les idées deviennent réalité en 1982. En effet le changement souhaité (l'avènement des intellectuels au pouvoir) se produit au Cameroun, six mois après la publication de l'ouvrage d'Aoué Tchany. En novembre 1982, on assiste comme au pays Kamadougou [Cameroun] au "désistement de El Hadj Salim Modiki [entendez $\mathrm{El} \mathrm{Hadj} \mathrm{A.} \mathrm{Ahidjo]} \mathrm{et} \mathrm{de} \mathrm{son} \mathrm{régime,} \mathrm{tyrannique} \mathrm{et} \mathrm{laxiste,} \mathrm{au} \mathrm{profit}$ d'un intellectuel intéressé par les valeurs humaines" [entendez Paul Biya].

\section{L'Après Ahidjo: Les intellectuels au pouvoir en 1982...}

Certains ont cru voir dans ce changement des lueurs d'espoir pour le Cameroun; "le printemps camerounais" était une chance pour le pays qui devait se démarquer de la plupart de ses rivaux africains, la Côte d'Ivoire en l'occurence, pour tirer un meilleur profit de ses ressources naturelles et humaines. Avec la nouvelle politique du "Renouveau: rigueur et moralisation ${ }^{446}$ proposée par le nouveau président, on voyait poindre à l'horizon un "renouveau intellectuel" capable d'éloigner le pays des voies du sous-développement, de la misère, de la pauvreté, bref, de la crise économique et sociale que commençait à vivre le Cameroun. 
La réalité aujourd'hui est tout autre si bien que certains n'hésitent pas à crier à "la trahison de l'intelligentsia"47. En effet, que constate-t-on en cette période de modernité? D'abord que comme le démontre la théorie de Gramsci, l'intelligentsia camerounaise ne constituent pas un espace autonome: la collusion des intellectuels avec le pouvoir, hier, médiane, voire discrète est aujourd'hui médiatique et médiatisée. Ensuite, l'intellectuel camerounais à quelques exceptions près, est généralement à la remorque du politique, soit sous la figure du Prince, soit sous celle de l'opposant. La politique (action politique) semble constituer son domaine de définition, le repère majeur de son identité. Ainsi par exemple, pour ne prendre que l'exemple des enseignants de l'Université de Yaoundé, on a l'impression que tout se passe comme si depuis leur intégration dans le corps enseignant de l'unique université du pays, ceux-ci, dans leur carrière, comme dans la préparation de leur retraite, fondent leur stratégie (de carrière) sur l'alliance ou non avec le Prince du moment. Ceci s'observe de plus en plus aujourd'hui avec l'entrée spectaculaire dans les différents gouvernements et dans les instances administratives et politiques dirigeantes de professeurs (politologues et juristes pour la plupart), d'enseignants d'université; de leur engagement militant auprès du Parti au pouvoir (le Rassemblement démocratique du peuple camerounais RDPC) ou dans les partis d'opposition; de leur participation dans les pseudo-comités ou commissions créés par le Gouvernement aux fins de définir la marche/démarche à suivre (définir une stratégie camerounaise) pour rentrer de manière originale dans l'ère démocratique.

En somme, Faute de forum démocratique, le débat politique et les discours sur la société et la Cité se déroulent dans les ouvrages littéraires. Les écrivains jouent ainsi leur rôle d'intellectuel. Même si ces auteurs savent que leurs ouvrages sont censurés au Cameroun ou saisi depuis la France comme Main basse sur le Cameroun de Mongo Beti, ils sont persuadés comme dit J. Ackad que s'ils écrivent et publient en français avant même de pouvoir atteindre la minorité africaine (de privilégiés) qui pourront les lire; ces romans servent à la diffusion à l'étranger, spécialement en France d' "idées politiques", entre autres, les partis politiques autres que celui au pouvoir n'étant pas admis dans les faits.

On constate que la plupart des "intellectuels" camerounais ont choisi de participer au projet hégémonique de l'Etat post-colonial, par intérêt personnel (jouir des avantages et privilèges) ou de bonne foi (volonté de combler le "vide" administratif laissé par le colonisateur et/ou tentative de réforme de l'intérieur). Nous constatons également qu' Ahidjo a réussi à réunir autour de lui "ses" intellectuels et à monter ces derniers contre les "autres" intellectuels, ceux qui étaient de l'autre bord, 
les opposants au projet hégémonique. Le contrôle du régime sur les intellectuels s'est alors réalisé de plusieurs manières: d'une part, par l'agitation et l'exhibition de prébendes offertes par le Gouvernement et le parti unique (la trappe), d'autre part par la répression (la traque) de ceux qui ne voulaient pas se "ranger" ou se "discipliner". Cette participation,"forcée" pour certains, peut dans un contexte de parti unique trouver des justifications -système de parti unique, intolérant à l'égard de toute formes de pluralisme, d'opinion dissidente ("subversive"), et de toute voie/voix discordante/dissonante-, elle peut même être humainement explicable comme illustre le cas des intellectuels de l'ex-RDA ${ }^{48}$. Peut-elle encore trouver des justifications similaires en période de modernité, de "regain démocratique", et surtout après l'avènement d'un intellectuel au pouvoir au Cameroun depuis 1982.

Sans pour autant vouloir justifier le sentiment de peur et d'insécurité que vivent la plupart des intellectuels camerounais et africains en général -sentiment tout aussi justifié- il nous semble que bon nombre d'entre eux devraient faire leur mea culpa et reconnaître humblement comme le font aujourd'hui les intellectuels d'Europe de l'Est qu'ils ont "manqué de courage, [qu'ils n'ont] pas su organiser une résistance solidaire. [Que] les Robert Havemann, AndreïSacharov ou Vaclav Havel sont restés solitaires dans leur opposition au pouvoir. Qu'ils ont, [les intellectuels] appartenu à cette intelligentsia qui a fonctionné en silence tout en étant opposés à un système dont [ils ont] seulement prolongé l'agonie"49. 


\section{NOTES}

1. - Birabuza André, Trahison des Clercs et renaissance noire en Afrique, Bujumbura, Editions Intore, 1994, 127 p.

2. - Comi M. Toulabor: " avant-propos " au numéro consacré au " intellectuels africains ", Politique africaine, $\mathrm{n}^{\circ} 51$, octobre 1993, p. 5.

3. - Yewah Emmanuel a montré la construction et la déconstruction des idéologies à travers l'analyse de la littérature camerounaise: “ Ideology and the De/Naturalization of Meaning in the Cameroonian Novel ", dans Afrika Focus, Vol. 9, nr. 3-4, 1993, pp. 179-192.

4. - Rossatanga-Rignault Guy: "l'insoutenable condition du clerc gabonais ", Politique africaine, $\mathrm{n}^{\circ} 51$, octobre 1993, pp. 48 et $\mathrm{s}$.

5. - Monga Célestin, Anthropologie de la colère. Société civile et démocratique en Afrique noire, Paris, L'Harmattan, 1994, 167p.

6. - Kamto Maurice, L'urgence de la pensée. Réflexions sur une précondition du développement en Afrique. Yaoundé, Editions Mandara, 1995, p. 110-111.

7. - Comi M. Toulabor, avant-propos, Politique africaine, $\mathrm{n}^{\circ}$ 51, octobre 1993, précité, p. 5

8. - Mongo Beti, Main basse sur le Cameroun. Paris, Maspéro, 1977 (1ere éd. 1972). 270 p. (cit. p. 134).

9. - Les causes profondes de ce phénomène ne semblent pas suffisamment soulignées par Augustin Oyowe, "La fuite de cervaux: des années d'investissement perdues pour les pays en développement", Le Courrier CEE/ACP, septembre-octobre 1996, p.59-60.

10. - Voir récemment encore, le cas du professeur Jean-Marc Ela, qui a dû fuir le pays, suite à d'innombrables brimades, humiliations et harcèlement, lire, Mbembe Achille:

“Jean-Marc Ela, exil forcée... ", Golias Magazine, n 44, septembre/octobre 1995, pp. 68-72. Il faut rappeler que le "confort" des pays occidentaux ne saurait remplacer pas la quête de dignité et de liberté auxquelles chaque homme aspire dans/pour son pays.

11. - F. Bon et M. A. Burnier, Les nouveaux intellectuels. Paris, Ed. du Seuil, 1975 (1ere éd. 1973).

12. - Nous remercions le lecteur anonyme de la revue Afrika Focus, de nous avoir conforté dans cette vision des choses et de nous avoir suggéré le bref historique qui suit.

13. - Mgr Tumi, dans Jeune Afrique Economie, 134, Août 1990. p.90. Mgr Tumi, actuellement archevêque de Douala, est le premier Cardinal du Cameroun (et le seul à l'heure actuelle) est un anglophone. Il est connu pour ses prise de position en faveur des droits de l'homme. 
14. - Si l'on en croit des spécialistes de la question, comme Javier Herrera, "la nature de la crise financière camerounaise et les mesures prises pour la combattre: faut-il ajuster les programmes d'ajustement structurel?", dans Georges Courade (dir), Le village camerounais à l'heure de l'ajustement, Paris, Karthala (coédition OCISCA), 1994, p.40-51.

15. - "Docta" ou "Docteur" sont des termes utilisés pour désigner les diplômés de l'enseignement supérieur, ou les intellectuels en général, et les étudiants en fin de second cycle ou en troisième cycle, en particulier.

16. - Pour la naissance du "Cameroun " et l'héritage que les allemands ont légués aux Camerounais, lire Owona Adalbert, Naissance du Cameroun 1884-1914. Paris, L'Harmattan, 1996, 236 p. (Coll. Racines du Présent).

17. - Voir essentiellement J.-F. Bayart, L'Etat au Cameroun. Paris, Presses FNSP, 1985 (2e éd. revue et augmentée) dont nous reprenons ici quelques idées et, L'Etat en Afrique: la politique du ventre. Paris, Fayard, 1990. 439 p. Lire également les travaux de J.-F. Médard sur le système politique camerounais (CEAN, Bordeaux). L'ouvrage de Samuel Eboua, proche collaborateur d'Ahidjo nous introduit également dans la logique de ce pouvoir: Ahidjo et la logique du pouvoir. Paris, L'Harmattan, 1995, 236 p. (Coll. Mémoires africaines).

18. - Notre intervention au Colloque du CEAN/IEP de Bordeaux en 1989 sur "les rapports entre l'administration et les populations centrales en Afrique". Colloque non publié. Voir notre compte-rendu rédigé avec la collaboration de J.-P. Daloz dans la Revue française d'Administration publique, $\mathrm{n}^{\circ} 56$, octobre-décembre 1990, pp 703-706.

19. - A. Ahidjo "discours de clôture du IVème Conseil national de l'UNC" L'unité, 616, 26 février 1977 (cité par J.-F. Bayart, L'Etat au Cameroun, op.cit. p.252).

20. - Une des thèses de J.-F. Bayart, ibidem.

21. - Kamto Maurice, L'urgence de la pensée..., précité, p. 111.

22. - Lire les propos du président de la République de Côte d'Ivoire cité par P. N'da, Les intellectuels et le pouvoir en Afrique noire. Paris, L'Harmattan, 1987, pp. 87-88.

23. - Mongo Beti, op. cit.

24. - J. F. Youbi "La camerounisation des cadres", mémoire de Licence, Faculté de Droit, Université de Yaoundé, juin 1974, 83 p.

25. - J. Owona, Droit administratif spécial de la république du Cameroun. Paris, EDICEF, 1985, p. 30.

26. - La situation est pire aujourd'hui, où l'on assiste à un démantellement quasi programmé des institutions universitaires (absence de moyens financiers et techniques, interdiction des activités syndicales, agressions, humiliations, délation à l'encontre des enseignants, nomination politique, etc., lire Mbembe Achille “ Jean-Marc Ela, exil forcée... ", Golias Magazine, n 44, septembre/octobre 1995, pp. 68-72.. 
27. - Le régime actuel au Cameroun semble s'appuyer sur des juristes et des politistes. Parmi ceux-ci, on citera parmi les ministres, les professeurs Joseph Owona, Augustin Kontchou Kemogne, Joseph-Marie Bipoun-Wum, tous, professeurs d'université et spécialistes de droit constitutionnel et de science politique. Ça peut aider en ces périodes de " démocratisation "!.

28. - Le Centre universitaire était constitué à l'époque de deux établissements: ENSA (Ecole nationale supérieure agronomique, qui comptait 250 étudiants) et l'ITA (Institut des Techniques agricoles, 540 étudiants). Ces fonctions, bien qu'incompatibles, permettaient à leurs détenteurs de contrôler plusieurs lieux de pouvoir à la fois au profit du Parti au pouvoir.

29. - P.-F. Ngayap, Cameroun qui gouverne ?. Paris, L'Harmattan, 1983.

30. - Ci-dessous la liste des auteurs cités par ordre alphabétique: Ouvrages d'auteurs camerounais cités dans l'article et classés par ordre alphabétique:

- J. C. Aoué Tchany, Du folklore en enfer. Paris, La pensée universelle, 1982, 210 p.

- F. Bebey, La poupée ashanti, Yaoundé, Ed. Clé, 1973, 221 p.

- R. Ekossono, Le soleil rouge.

- Ela Jean-Marc, Le cri de l'homme africain, Paris, Karthala, 1980.

- Ela Jean-Marc, L'Afrique des villages: la riposte paysanne, Paris, Karthala, 1982.

- D. Etounga-Manguelle, La colline du fromager. Yaoundé, Ed. Clé, 1979.

- F.-B. M. Evembé, Sur la terre en passant. Paris, Présence africaine, 1966, 112 p.

- D. Ewandé, Vive le Président! La fête africaine. Paris, Albin Michel, 1968, 224

p. Réedité chez L'Harmattan, 1984. 222 p. (Collection Encres noires).

- J. Ikelle Matiba, Cette Afrique-là, Paris, Présence Adricaine, 1962, 241 p.

- Y. Karone, Le bal des caïmans. Paris, Karthala, 1980 et Nègre de paille. Paris, Ed. Silex, 1982.

- B. Matip, Laisse-nous bâtir une Afrique debout, Ed. Afriscascope. 1979.

- Ch.-G. Mbock: Quand saigne le palmier. Yaoundé, Ed. Clé, 1978, 144 p. et La croix du coeur. Yaoundé, Ed. Clé, 1984, 228 p.

- R. G. Medou Mvomo, Afrika Ba'a. Yaoundé, Ed. Clé, 1969, 181 p.

- Mongo Beti, Main basse sur le Cameroun: autopsie d'une décolonisation. Paris, Maspéro, 1977 (1ere éd. 1972). Mongo Beti a publié d'autres ouvrages politiques dont:

- Remember Ruben. Paris, UGE. 1974 (Collection 10/18).

- Perpétue et l'habitude du malheur. Paris, Ed. Buchet-Chastel, 1974.

- La ruine presque cocasse d'un polichinelle (Remember Ruben II). Paris, Ed. Peuples Noirs. 1979, 320 p.

- B. Nanga, Les chauves souris. Paris, Présence africaine, 1980.

- A. Ngongwikuo, Taboo Love. New York, Hicksville, Exposition Press. 1980.

- J. Oto, Drame d'un pays. Yaoundé, Ed. Clé. 1979.

- J. Owono, Tante Bella. Yaoundé, Au Messager, 1959, 259 p.

- Pabe Mongo, Bogam Woup: Allégorie de la mutation. Yaoundé, Ed. Clé, 1960.

- R. Philombè, Choc anti-choc. Yaoundé, Semences africaines, 1978, 40 p.

- Zanga Tsogo, Vies de femmes. Yaoundé, Ed. Clé, 1982, 121 p. 
31. - Exemple cité par J.-F. Bayart, L'Etat au Cameroun, op.cit. p.102. Citation extraite de la revue camerounaise, 16 novembre 1963, p.81.

32. - Cité par Mongo Beti, Main basse sur le Cameroun, op. cit. pp. 201 et s.

33. - Lire Mongo Beti, op. cit. pp. 96 et s. Sur la DIREDOC, lire le même ouvrage pp. 109-110. Il faut noter que les principaux camps d'internement sont ceux de Yoko (Mbam), Mantoum (Bamoun), Tcholliré (Bénoué) et Mokolo (Margui-Wandala). D'autre part, il est reconnu que même les postes diplomatiques sont infestés d'agents de la DIREDOC qui sont chargés de rendre compte de tout acte de subversion des camerounais émigrés contre le président de la République et son régime.

34. - Déclaration dans le journal catholique français La Croix, le 15 janvier 1963.

35. - G. Chaffard, Les carnets secrets de la décolonisation. Paris, Calman Lévy, 1965 et 1967, t. et t.II. Cit. t. II, p. 347.

36. - Voir le récent ouvrage d'Achille Mbembe consacrée à Um Nyobè, publié à Paris chez Karthala en 1996.

37. - Il est cependant vrai de constater, qu'intellectuel ou non, Um Nyobè était l'homme à abattre.

38. - Citer l'article du Messager sur Osendé Afana.

39. - Josette Ackad, Le roman camerounais et la critique. Paris, Ed. Silex, 1985.

40. - Ironie qui frise la dérision dans le sens étudié par Comi Toulabor pour le cas du Togo sous Eyadéma: "L'énonciation du pouvoir et de la richesse chez les jeunes "conjoncturés" de Lomé (Togo)", Revue française de science politique, vol. $35, \mathrm{n}^{\circ} 3$, juin 1985, ou du même auteur, Le Togo sous Eyadéma, Paris, Karthala, 1986, 340 p.

41. - Bien que l'ouvrage de Jean-Marc Ela n'est pas repris dans l'analyse des critiques littéraires (Ackad et Ndachi Tagne, que nous avons utilisés), son ouvrage Le cri de l'homme africain, paru aux Editions L'Harmattan en 1980, mérite d'être plus connu et étudié. Car, il trace les marques d'un engagement qui conduit ce courageux intellectuel, théologien et sociologue africain, à l'exil vers le Canada depuis le 6 août 1995.

42. - J. Ackad, op. cit. p. 22.

43. - Cf. Note 5, supra.

44. - D. Ndachi Tagne, Roman et réalités camerounaises. Paris, L'Harmattan, 1986, 301 p.

45. - D. Ndachi Tagne, Roman et réalités camerounaises, précité, p. 222.

46. - Paul Biya, Pour le libéralisme communautaire. Lausanne/Paris, Ed. P.-M. Fabre/ABC, 1986, 158p. 
47. - A. Kom "Trahison d'une intelligentsia", Jeune Afrique Economie, $n^{\circ} 165$, mars 1993, pp. 122-123.

48. - Cité dans l'article de Brigitte Pätzold, journaliste: "la recherche et les aveux:

Intellectuels Est allemands sur la selette", Le Monde diplomatique, Avril 1993, p.11.

49. - Jens Reich (Chercheur à l'Institut de Biologie moléculaire de Berlin In: Abscheid von den Lebenslügen (Adieu aux mensonges d'une vie), Rowcht, Berlin, 1992 p. 176. Cité par B. Pätzold, art. précité. 